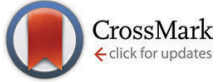

Cite this: Phys. Chem. Chem. Phys., 2016, 18, 5938

Received 27th August 2015, Accepted 4th December 2015

DOI: $10.1039 / c 5 c p 05105 c$

www.rsc.org/pccp

\section{The nature of the silicaphilic fluorescence of PDMPO $\uparrow$}

\author{
Mithun Parambath, ${ }^{a}$ Quentin S. Hanley, ${ }^{a}$ Francisco J. Martin-Martinez, ${ }^{b}$ \\ Tristan Giesa, ${ }^{b}$ Markus J. Buehler ${ }^{b}$ and Carole C. Perry*a
}

\begin{abstract}
PDMPO (2-(4-pyridyl)-5-((4-(2-dimethylaminoethylaminocarbamoyl)methoxy)phenyl)oxazole), has unique silica specific fluorescence and is used in biology to understand biosilicification. This 'silicaphilic' fluorescence is not well understood nor is the response to local environmental variables like solvent and $\mathrm{pH}$. We investigated PDMPO in a range of environments: using UV-vis and fluorescence spectroscopy supported by computational data, (SPARC, molecular dynamics simulations, density functional theory calculations), dynamic light scattering and zeta potential measurements to understand the PDMPO-silica interaction. From absorption data, PDMPO exhibited a $\mathrm{p} K_{\mathrm{a}}$ of 4.20 for $\mathrm{PDMPOH}_{2}{ }^{2+}$ to $\mathrm{PDMPOH}^{+}$. Fluorescence emission measurements revealed large shifts in excited state $p K_{a}{ }^{*}$ values with different behaviour when bound to silica ( $p K_{a}{ }^{*}$ of 10.4). PDMPO bound to silica particles is located in the Stern layer with the dye exhibiting $\mathrm{pH}$ dependent depolarising motion. In aqueous solution, PDMPO showed strong chromaticity with correlation between the maximum emission wavelength for $\mathrm{PDMPOH}^{+\star}$ and dielectric constant (4.8-80). Additional chromatic effects were attributed to changes in solvent accessible surface area. Chromatic effects were also observed for silica bound dye which allow its use as a direct probe of bulk pH over a range far in excess of what is possible for the dye alone (3-5.2). The unique combination of chromaticity and excited state dynamics allows PDMPO to monitor pH from 3 to 13 while also reporting on surface environment opening a new frontier in the quantitative understanding of (bio)silicification.
\end{abstract}

\section{Introduction}

There is considerable interest in the unique fluorescence of PDMPO (2-(4-pyridyl)-5-((4(2-dimethylaminoethylaminocarbamoyl)methoxy)phenyl)oxazole) in the presence of silica and its resulting use as a silica probe. ${ }^{1}$ PDMPO has been widely used to study new silica deposition. Examples include: precipitation of silica in diatoms, ${ }^{2,3}$ silica deposition in Equisetum arvense, ${ }^{4}$ observation of the skeletal growth patterns in Polycystine radiolarians, ${ }^{4}$ and identifying silica transporters in silicifying organisms ${ }^{5}$ such as Bolidophyceae and Parmales. ${ }^{6}$ There have been many attempts to develop probes for studying the molecular properties of silica using a variety of molecules including rhodamine $\mathrm{B},{ }^{7}$ rhodamine $19,^{7}$ rhodamine $101,{ }^{7}$ rhodamine $123,{ }^{8}$ bis(cyclopentadienyl) titanium dichloride, ${ }^{9}$ and fluorescein coupled via 3-aminopropyl triethoxysilane, ${ }^{10}$ but these alternatives have a range of problems such as low accumulation efficiency and insolubility in aqueous solution. PDMPO has proven to be a very effective tracer during

\footnotetext{
${ }^{a}$ School of Science and Technology, Nottingham Trent University, Nottingham, NG118NS, UK. E-mail: Carole.Perry@ntu.ac.uk

${ }^{b}$ Department of Civil and Environmental Engineering,

Massachusetts Institute of Technology, USA

$\dagger$ Electronic supplementary information (ESI) available. See DOI: 10.1039/ c5cp05105c
}

biosilicification and has seen wide application in silica related studies. pH changes inside acidic organelles. ${ }^{11}$ A further class of oxazole dyes derived from 2,5-diphenyloxazole are known to exhibit unique solvatochromic and electron transfer properties. ${ }^{12}$ These dyes are weakly basic amines that selectively accumulate in cellular compartments with low internal $\mathrm{pH}$ making them valuable tools to investigate the biosynthesis and pathogenesis of lysosomes. ${ }^{13}$ Oxazole dyes can freely permeate cell membranes and concentrate in acidic organelles and PDMPO has been used to study $\mathrm{pH}$ at high hydrostatic pressure. ${ }^{14}$ Shimizu et al. first demonstrated a "silica-philic" fluorescence from PDMPO at $510 \mathrm{~nm}$ and noted its unique properties as a silica tracer. ${ }^{1}$ Although PDMPO-silica interactions are being used by scientists, ${ }^{1,3-5,15-17}$ there are gaps in our understanding. Specifically: the mechanism of the PDMPO-silica interaction is unknown; the spectroscopic details are unclear leading to difficulty selecting optimal excitation and emission wavelengths; the process creating "silicaphilic" fluorescence in PDMPO is unknown; the location of PDMPO on silica is not known; and while proposals have been made to use PDMPO to study silica polymerisation, experimental results to date were not specific enough to correlate PDMPO behaviour with the degree of oligomerization.
PDMPO is an oxazole dye developed to study intracellular 
This study investigates the unique fluorescence of PDMPO in the presence of silica. We began by identifying the possible protonation states of PDMPO and estimated their $\mathrm{p} K_{\mathrm{a}}$ 's by computational methods. To support the experimental results, we performed Density Functional Theory (DFT) calculations to characterize the reactivity and protonation of the different species under study. The Fukui function was used to explain the behaviour of putative protonation sites. Additionally, the solvent accessible surface area (SASA), which is closely related to chromatic effects on fluorescence, was estimated from DFT and molecular dynamics (MD) simulations. We used these considerations to inform a set of experiments investigating the ground and excited state behaviour of PDMPO beginning in aqueous solutions, proceeding through a range of solvents, and finishing with silica materials (Stöber particles $(50 \mathrm{~nm})$ ). These data provide a clear understanding of the silica dye interaction and provide a significant analytical framework for application of this technique in biology and chemistry.

\section{Materials and methods}

\subsection{Materials}

PDMPO (LysoSensor ${ }^{\mathrm{TM}}$ yellow/blue DND-160, 1 mM in dimethylsulfoxide) was obtained from Life Technologies. Chemicals for preparation of citrate and phosphate buffers, ammonia solutions, tetraethoxysilane (TEOS), coumarin 153, tryptophan, HPLC grade water, were obtained from Sigma Aldrich. Other solvents (water, methanol, ethanol, acetone, acetonitrile, dimethylsulfoxide, chloroform) were purchased from Fisher Scientific. When required, solutions were prepared in HPLC grade water.

\subsection{Predictive modelling of speciation}

Predictive chemical modelling of the $\mathrm{pH}$ dependent chemical forms of PDMPO and the associated $\mathrm{p} K_{\mathrm{a}}$ 's was performed using the web version of SPARC. ${ }^{18}$ The SPARC computational approach is based on the combination of well-established structure activity relationships, ${ }^{19}$ linear free energy relationships ${ }^{20}$ and perturbed molecular orbital theory ${ }^{21}$ to estimate $\mathrm{p} K_{\mathrm{a}}$. The SPARC method of $\mathrm{p} K_{\mathrm{a}}$ determination has been tested on 3685 compounds to estimate 4300 ionization $\mathrm{p} K_{\mathrm{a}}$ 's and their overall RMS deviation from measured values was $0.37 .^{18}$

\subsection{Computational methods}

Density functional theory (DFT) calculations optimizing the molecular geometries and calculations of the electronic structure of the $\mathrm{pH}$ relevant PDMPO species were performed using the ORCA package ${ }^{22}$ with the B3LYP/6-31G* level of theory. ${ }^{23,24}$ The reactivity of the PDMPO species was characterized using the Fukui function dual descriptor within the framework of conceptual DFT. ${ }^{25}$

Molecular dynamics (MD) simulations were performed to minimize the molecules using 1 nanosecond simulations with the LAMMPS MD software ${ }^{26}$ in an isochoric-isothermal ensemble at $300 \mathrm{~K}$ using the CVFF force field and a time step of $0.1 \mathrm{fs}$.
The partial charges on the atoms were taken from DFT calculations (ChelPG model) and ions added to neutralize the system.

The solvent accessible surface area (SASA) of the system was calculated from the molecular dynamics trajectory and the minimized DFT structures using the SASA tool in $\mathrm{VMD}^{27}$ using an indenter of $1.4 \AA$.

\subsection{Absorbance spectroscopy}

All spectroscopy experiments were carried out in $0.1 \mathrm{M}$ phosphate at $\mathrm{pH} \geq 5$ and citrate $\mathrm{pH} \leq 5$ buffers with some experiments in the range $\mathrm{pH}$ 4-6 carried out in both buffers to ensure no effect of buffer identity on the fluorescence behaviour observed. To correct for small variations in concentration and some interference from DMSO the spectra were normalised relative to the isobestic point (352 $\mathrm{nm}$ ) and analysis restricted to 250-450 $\mathrm{nm}$. Absorption spectroscopy was carried out in a dual beam spectrometer (JASCO V-670) in $1 \mathrm{~cm}$ quartz cuvettes over a $\mathrm{pH}$ range 2.5-12.01 with all samples prepared in $0.1 \mathrm{M}$ buffer with PDMPO added to give a concentration of $4.0 \times 10^{-5} \mathrm{M}$.

\subsection{Fitting $\mathrm{p} K_{\mathrm{a}} \mathrm{s}$ to spectroscopic data}

The DATAN software tool developed by Kubista et al. ${ }^{28,29}$ was used to calculate spectral profiles, concentrations and equilibrium constants by utilizing equilibrium expressions that are related to the components. In this study, all the recorded absorbance data and fluorescence data were assembled into data matrices and introduced to the DATAN programme ${ }^{29}$ to obtain pure spectra for the individual species, concentration profiles and acidity constants of the dye in absorbance and fluorescence modes.

\subsection{Fluorescence spectroscopic properties of PDMPO}

Fluorescence measurements were carried out in 96-well plates (Nunc optical bottom plates (164588)) using a commercial microplate reader (M200 pro; Tecan). Corrected spectra were computed over the range 300-678 nm using standard methods ${ }^{30}$ relative to tryptophan in water (300-428 $\mathrm{nm}$ with $400 \mathrm{~nm}$ excitation) and coumarin 153 in methanol (486-678 nm with $402 \mathrm{~nm}$ excitation) with interpolated values between 428 and $486 \mathrm{~nm}$. The effect of acid/base on the fluorescence emission of protonated/ non-protonated states of the dye $\left(1.0 \times 10^{-7} \mathrm{M}\right)$ was studied in buffers with $\mathrm{pH}$ ranging from 2.3 to 14 . The wavelength for excitation was chosen as $360 \mathrm{~nm}$ for maximum response which is close to the isobestic point $(352 \mathrm{~nm})$ of the $\mathrm{PDMPOH}_{2}{ }^{2+}$ to $\mathrm{PDMPOH}^{+}$transition of the dye.

\subsection{Solvatochromic properties of PDMPO}

The effect of solvent polarity on the fluorescence emission of the dye $\left(1.0 \times 10^{-7} \mathrm{M}\right)$ was studied in protic solvents (water, ethanol, acetone), polar aprotic solvents (DMSO and acetonitrile), and non-polar solvents (chloroform) having a range of dielectric constants. A series of binary mixtures of ethanol and water $(\mathrm{pH}$ 7.0) and acetonitrile in water containing $0.1 \mu \mathrm{M}$ PDMPO was used to measure its behaviour in protic solvents over a range of dielectric constants. 


\subsection{Fluorescence emission of PDMPO on silica}

Silica nanoparticles (SNPs) were prepared using a modified Stöber process. ${ }^{31}$ Briefly, synthesis involved mixing two solutions (A and B) previously prepared with particle size adjusted by varying the concentration of ammonia in solution A. For $50 \mathrm{~nm}$ silica particles: solution A was prepared by taking $21.6 \mathrm{ml}$ of $0.2 \mathrm{M}$ ammonium hydroxide $\left(\mathrm{NH}_{4} \mathrm{OH}\right)$ in distilled deionized water $\left(\mathrm{ddH}_{2} \mathrm{O}\right)$ and adding ethanol to make up the volume to $80 \mathrm{ml}$ in ethanol. Solution B was prepared by diluting $22.3 \mathrm{ml}$ of TEOS in ethanol to $76.6 \mathrm{ml}$. Freshly prepared solutions were heated to $50{ }^{\circ} \mathrm{C}$, mixed then stirred at $4000 \mathrm{rpm}$ for 2 hours at the same temperature before allowing them to mature at room temperature for 1 day. Ethanol was removed by rotary evaporation at $50{ }^{\circ} \mathrm{C}$, the sample centrifuged at $4000 \mathrm{rpm}$ for 10 minutes and the particles washed with ethanol then distilled water and freeze dried.

Solutions containing the dye at $0.1 \mu \mathrm{M}$ were prepared in $0.1 \mathrm{M}$ buffers (pH 1.8 to 13.8). Silica nanoparticles (50 nm diameter, final concentration: $1 \mathrm{mg} \mathrm{ml}^{-1}$ ) were suspended in buffer solutions, incubated for 30 minutes at $25{ }^{\circ} \mathrm{C}$ on a plate shaker at $10 \mathrm{rpm}$ before measuring changes in the fluorescence emission spectrum. An excitation wavelength of $360 \mathrm{~nm}$ was used to collect fluorescence emission spectra for all silica PDMPO interactions.

\subsection{PDMPO-silica interactions}

Experiments were conducted in $0.1 \mathrm{M}$ buffer ( $\mathrm{pH} 1.8$ to 13.8) with PDMPO $(0.1 \mu \mathrm{M})$ and silica particles $(50 \mathrm{~nm})$ at a nominal concentration of $1 \mathrm{mg} \mathrm{ml}^{-1}$. Zeta potential and dynamic light scattering (DLS) measurements were carried out at $25{ }^{\circ} \mathrm{C}$. The hydrodynamic diameter $\left(D_{\mathrm{h}}\right)$ of Stöber particles in the presence and absence of PDMPO was calculated as an average of five independent measurements.

Concentration dependent effects of the dye were studied using a constant amount of SNP (50 nm diameter, $1 \mathrm{mg} \mathrm{ml}^{-1}$ ), varying the amount of dye present $(0.1 \mu \mathrm{M}$ to $800 \mu \mathrm{M})$. In a typical experiment (e.g. for $10 \mu \mathrm{M}$ dye) $500 \mu \mathrm{g}$ of SNP was added to $495 \mu \mathrm{l}$ buffer (phosphate at $\mathrm{pH} \geq 5$ and citrate at $\mathrm{pH} \leq 5$ ), sonicated for 1 hour and $5 \mu$ of $1.0 \mathrm{mM}$ dye was added and shaken vigorously. Samples were left to equilibrate for 30 minutes at $25{ }^{\circ} \mathrm{C}$ on a shaker at $10 \mathrm{rpm}$ before performing zeta potential measurement followed immediately by fluorescence emission measurements.

The fluorescence anisotropy of the dye in combination with silica was measured using SNPs (50 nm diameter, $1 \mathrm{mg} \mathrm{ml}^{-1}$ ) in $0.1 \mathrm{M}$ Buffer (pH 1.8 to 14.0$)$ with PDMPO $(0.1 \mu \mathrm{M})$. Steady state anisotropies were measured using a Tecan F200 with excitation filter 360/35 $\mathrm{nm}$ and emission filter 540/35 $\mathrm{nm}$. The PMT gain setting was 40 and the $G$ factor 1.256 .

2.9.1 pH dependent behaviour of PDMPO adsorbed on silica. Silica particles (50 $\mathrm{nm}$ diameter, $10 \mathrm{mg} \mathrm{ml}^{-1}$ ) were used and $\mathrm{pH}$ measurements performed in buffer $(0.1 \mathrm{M})$ in the presence of $1 \mu \mathrm{M}$ PDMPO. pH was measured before and after spectroscopic measurements. As for other experiments, samples were left to equilibrate for 30 minutes at $25{ }^{\circ} \mathrm{C}$ on a shaker at
$10 \mathrm{rpm}$. For fluorescence measurements the dye was excited at $360 \mathrm{~nm}$.

2.9.2 Fitting peak positions and relative areas. Spectra were decomposed by least squares minimisation of a two component Gaussian model after conversion to wavenumber. Parameters fitted were: peak position $(\mu)$, peak width $(\sigma)$, and scale (conversion factor to arbitrary fluorescence units).

\section{Results}

\subsection{Computational speciation studies}

To understand the effect of $\mathrm{pH}$ on the ground states of PDMPO, $\mathrm{p} K_{\mathrm{a}} \mathrm{s}$ were estimated computationally to provide an interpretive framework for subsequent UV-vis spectroscopic studies. The possible species are represented in Scheme 1. Although convention gives that the first proton lost from a compound gives rise to $\mathrm{p} K_{1}$, in this study we chose to omit the first two acidity constants from our discussions of the dye as they have no physical meaning in normal aqueous solutions. The SPARC computation predicted one neutral and four protonated states. Of these, three species are important above pH 1.2 (highlighted; Scheme 1); these are $\mathrm{PDMPOH}_{2}{ }^{2+}$ (predicted $\mathrm{p} K_{\mathrm{a} 1}$ 4.87 , protonation of the pyridine group and terminal amine), $\mathrm{PDMPOH}^{+}$(predicted $\mathrm{p} K_{\mathrm{a} 2}$ 6.78, protonation of terminal amine), and the neutral species, PDMPO.

Chemical species identified by SPARC and their estimated $\mathrm{p} K_{\mathrm{a}} \mathrm{s}$. Highlighted species are important in aqueous solution; the others are destroyed by acid hydrolysis. To reinforce the SPARC results, the Fukui function was used to estimate possible protonation sites and donor-acceptor behavior between the pyridine moiety and the phenyl ether group (Fig. 1) for the three

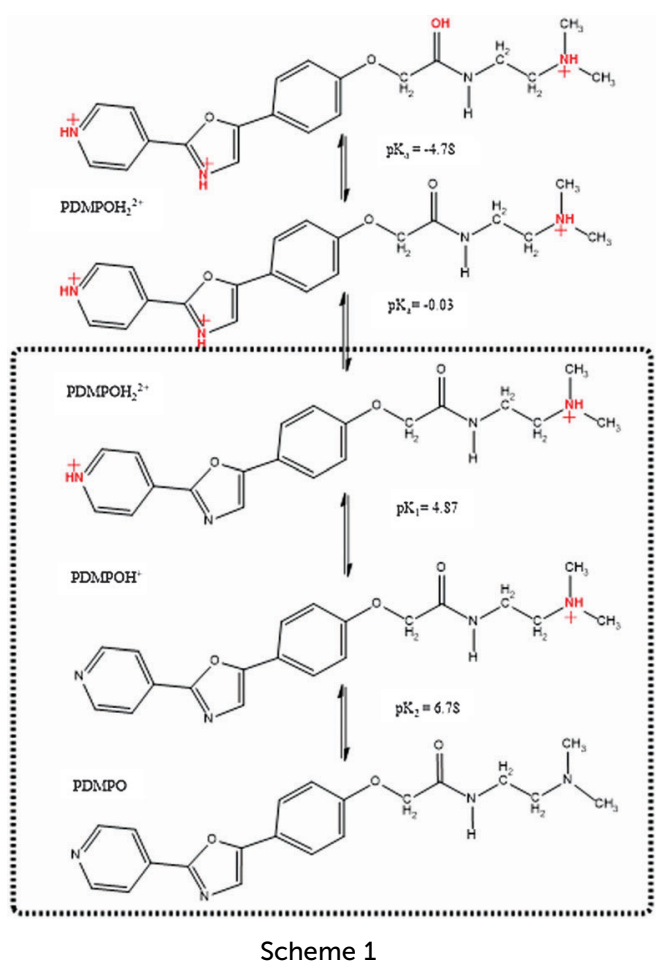




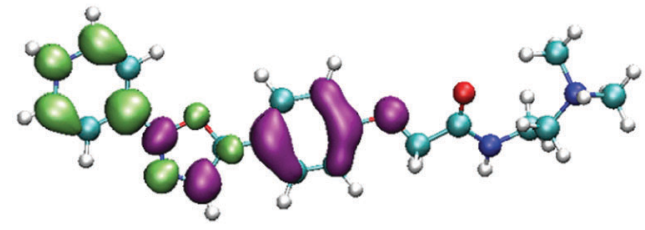

b
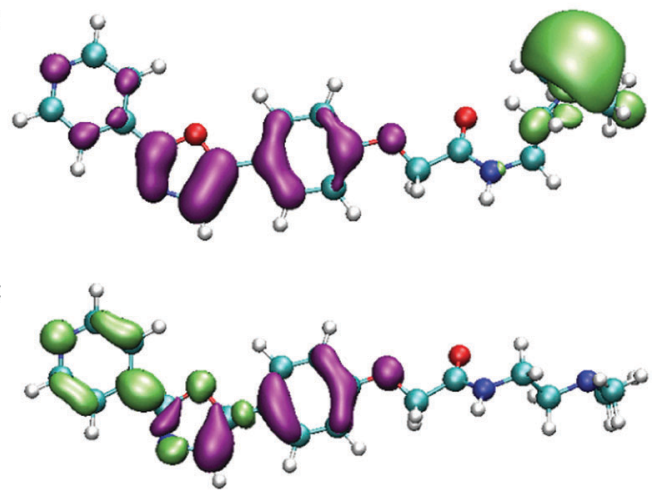

Fig. 1 Representation of the dual descriptor for the Fukui function. Green indicates regions that may undergo electrophilic attack (basic regions). Purple indicates regions that may undergo nucleophilic attack (acidic) (a) $\mathrm{PDMPOH}_{2}{ }^{2+}$ (b) $\mathrm{PDMPOH}^{+}$(c) PDMPO.

highlighted species in Scheme 1. The Fukui function describes how electron density changes in response to an increase or a decrease in the number of electrons in a molecule. The function is able to predict the preferred site for either a nucleophilic or electrophilic attack and is sometimes used to rationalize chemical reactivity principles such as the hard and soft acids and bases principle (HSAB), and therefore to understand protonation. $^{32}$ The localization of the Fukui function in PDMPO (Fig. 2c) indicates that the nitrogen in the pyridine and the oxygen of the phenyl ether part of the molecule have opposite behavior.

While the nitrogen is suitable for protonation (green in Fig. 1c), the oxygen becomes electrophilic (purple in Fig. 1c). This is consistent with the donor-acceptor design of the molecule. The calculated HOMO-LUMO energy gap decreased with increasing protonation (PDMPO (3.83 eV), $\mathrm{PDMPOH}^{+}$ $(3.58 \mathrm{eV})$ and $\mathrm{PDMPOH}^{2+}(2.66 \mathrm{eV})$ in broad agreement with experiment, see below.

\subsection{Absorbance properties of PDMPO}

The absorption spectra of PDMPO were measured in buffered solutions and normalised to an estimated isobestic point of $352 \mathrm{~nm}$ (Fig. 2a). DATAN analysis recovered only two of the species predicted by SPARC (nominally $\mathrm{PDMPOH}_{2}{ }^{2+}, \mathrm{PDMPOH}^{+}$) (Fig. 2b) with their concentrations and associated $\mathrm{p} K_{\mathrm{a}}$ (Fig. 2c). The recovered $\mathrm{p} K_{\mathrm{a}}$ (4.20) was in reasonable agreement with SPARC predictions (4.87) and previous reports. ${ }^{33}$ The absence of a $\mathrm{p} K_{\mathrm{a}}$ for $\mathrm{PDMPOH}^{+} / \mathrm{PDMPO}$ indicated the absorption spectra are dominated by protonation of the pyridine group and the protonation state of the amine side chain has negligible effect.

This was confirmed in the raw data (Fig. 2d) which showed no change in the absorbance at $331 \mathrm{~nm}$ above $\mathrm{pH}$ 6. This analysis a

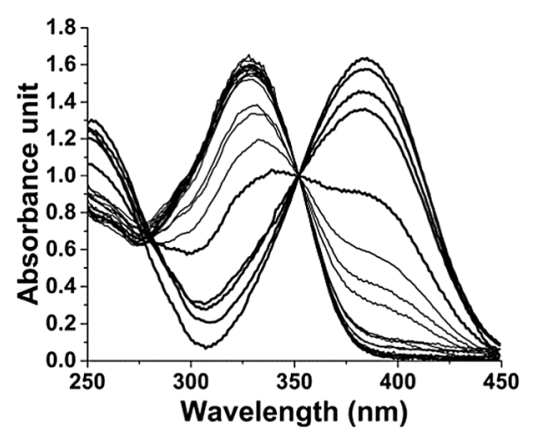

b

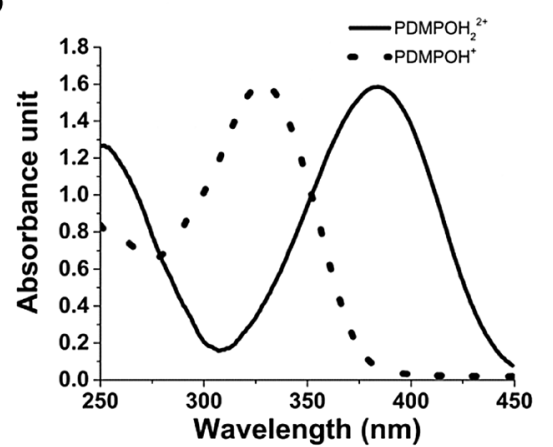

C

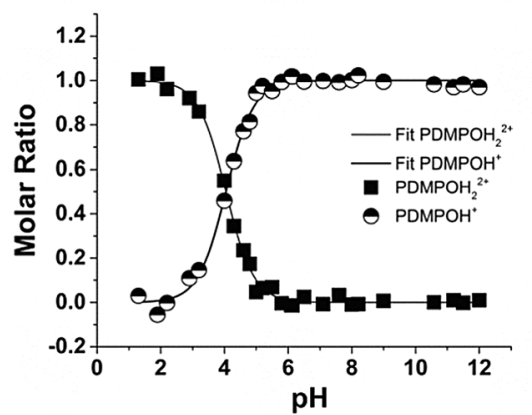

d

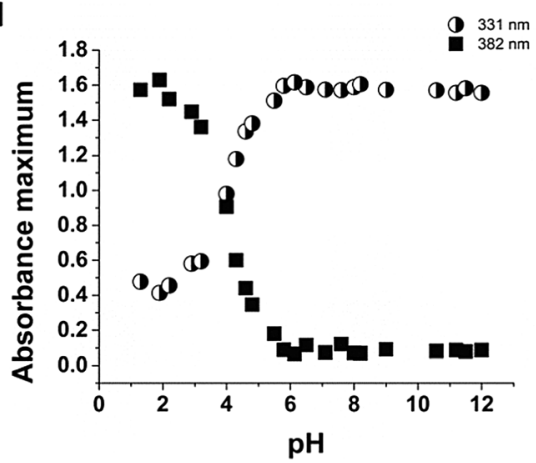

Fig. 2 Absorption spectra of PDMPO $(40 \mu \mathrm{M})$ in $0.1 \mathrm{M}$ buffers: (a) spectra recorded over the range $\mathrm{pH} 1.6$ to 12.8 ; (b) spectra of $\mathrm{PDMPOH}_{2}{ }^{2+}$ and $\mathrm{PDMPOH}^{+}$recovered by DATAN analysis; (c) calculated mole ratio of $\mathrm{PDMPOH}_{2}{ }^{2+}$ and $\mathrm{PDMPOH}^{+}$compared to those predicted by the resulting $K_{\mathrm{a}}$; (d) absorbance at $331 \mathrm{~nm}$ (nominally $\mathrm{PDMPOH}^{+}$) and $382 \mathrm{~nm}$ $\left(\mathrm{PDMPOH}_{2}{ }^{2+}\right.$ ) above $\mathrm{pH} 6$ there is no change in absorbance at $331 \mathrm{~nm}$.

left two species (Fig. 2b) with $\lambda_{\max }$ values at $382 \mathrm{~nm}(\varepsilon=3.84 \times$ $\left.104 \mathrm{M}^{-1} \mathrm{~cm}^{-1}\right)$ and $331 \mathrm{~nm}\left(\varepsilon=1.67 \times 10^{4} \mathrm{M}^{-1} \mathrm{~cm}^{-1}\right)$. Although protonation of the amine side chain has no role in the 
absorbance behaviour of the dye, it can affect the binding interaction of the dye with silica (see below). For clarity, the recovered species with $\lambda_{\max }$ at $331 \mathrm{~nm}$ will be referred to as $\mathrm{PDMPOH}^{+}$, however, at high $\mathrm{pH}$ significant amounts of the neutral species will be present. Attempts were made to determine the ground state $\mathrm{p} K_{\mathrm{a}}$ of PDMPO in the presence of silica. These measurements were unfeasible due to the requirement for low concentrations of the dye and the high scattering of the nanoparticulate solutions.

\subsection{Fluorescence spectroscopic properties of PDMPO in aqueous solutions}

Emission spectra of PDMPO in buffered aqueous solutions revealed $\mathrm{pH}$ dependent behaviour (Fig. 3a) with two distinct peaks over the $\mathrm{pH}$ range studied. DATAN analysis of the spectra (Fig. $3 \mathrm{~b}$ and c) suggested 3 forms with estimated excited state $\mathrm{p} K_{\mathrm{a}}{ }^{*} \mathrm{~S}$ of 4.2 and 13.7. The ground state $\mathrm{p} K_{\mathrm{a} 1}$ and first excited state $\mathrm{p} K_{\mathrm{a}}{ }^{*}$ appear to be the same (4.20) however, work with similar dyes in this class ${ }^{26}$ indicates they undergo excited state proton transfer from water to dye*. The "species" dominating between 4.2 and 13.7 (Fig. 3c) is a rate determined mixture of the protonated and deprotonated pyridine forms with a yield dependent on $\left[\mathrm{H}_{2} \mathrm{O}\right]$. As the $\mathrm{pH}$ approaches $\mathrm{p} K_{\mathrm{w}}$, the concentration of water decreases significantly due to formation of hydroxide ion and proton transfer becomes less favourable. The second recovered $\mathrm{p} K_{\mathrm{a}}{ }^{*} \mathrm{~S}(13.7)$ we ascribe to $\mathrm{p} K_{\mathrm{w}}$ rather than the actual $\mathrm{p}_{\mathrm{a}}{ }^{*}\left(\mathrm{PDMPOH}_{2}{ }^{2+}{ }^{*} / \mathrm{PDMPOH}^{*}\right)$ which is unobservable. To estimate $\mathrm{p} K_{\mathrm{a}}{ }^{*}\left(\mathrm{PDMPOH}_{2}{ }^{2+} / \mathrm{PDMPOH}^{*}\right)$ we applied the Förster equation ${ }^{24}$ which gave a value of 12.6. We believe our $\mathrm{pH}$ dependent spectroscopic determination is more definitive and better matches the expectations from work on similar molecules ${ }^{30}$ that also found $\mathrm{p} K_{\mathrm{a}}{ }^{*} \mathrm{~s}$ near 14 .

Similar to the absorbance behaviour, there was no evidence the ammonium group on the side chain influences the excited state and the recovered species with a peak at $454 \mathrm{~nm}$ will be referred to as $\mathrm{PDMPOH}^{+}$. This analysis leaves two excited state forms, $\mathrm{PDMPOH}_{2}{ }^{2+}{ }^{*}$ (protonated pyridine group; $\lambda_{\text {max }, \mathrm{em}}=$ $544 \mathrm{~nm}$ ) and $\mathrm{PDMPOH}^{+}$(neutral pyridine group; $\lambda_{\text {max,em }}=$ $454 \mathrm{~nm}$ ), which exist in three $\mathrm{pH}$ regions: (i) $\mathrm{pH}<4.2$ (dominated by $\mathrm{PDMPOH}_{2}{ }^{2+}$ ); $\mathrm{pH}$ 4.2-13.7 $\left(\mathrm{PDMPOH}^{+}\right.$and $\mathrm{PDMPOH}_{2}{ }^{2+*}$ in a kinetically defined ratio); and 13.7 $\left(\mathrm{PDMPOH}^{+}\right.$and $\mathrm{PDMPOH}_{2}{ }^{2+}$ in a kinetically defined ratio); and $\mathrm{pH}>13.7$ (dominated by $\mathrm{PDMPOH}^{+}$) (Scheme 2). The slight blue shift in the emission maxima between $\mathrm{pH} 4.2$ and 13.7 is consistent with a slight change in solvent accessible surface area between the neutral and singly protonated states (Table 1). The Lippert-Mataga equation ${ }^{34}$ relates solvatochromism to the solvent accessible surface area $S$ which we estimated calculated from DFT and MD. The energy difference between the ground state and the excited state scales with $1 / S^{3}$. The calculated surface areas for the three species are shown in Table 1. The decrease in the more protonated species is in agreement with the observed blue shift in the fluorescence spectrum. The DATAN analysis found only a single species between $\mathrm{pH} 4.3$ and 13.7 which represents the average of several forms. This results in a a

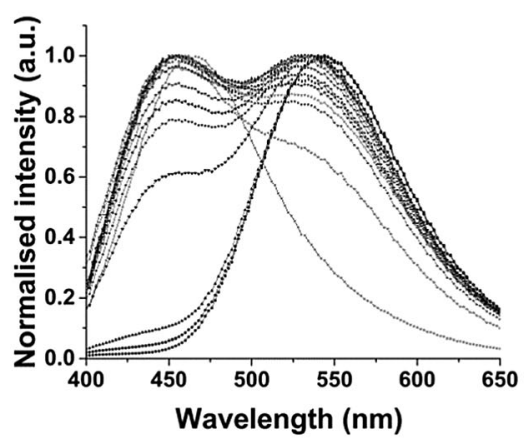

b

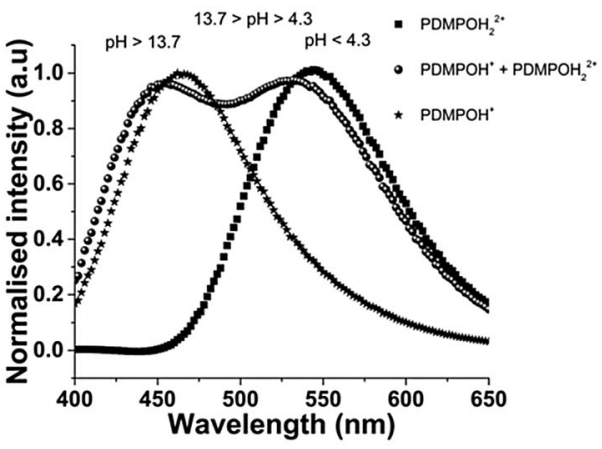

C

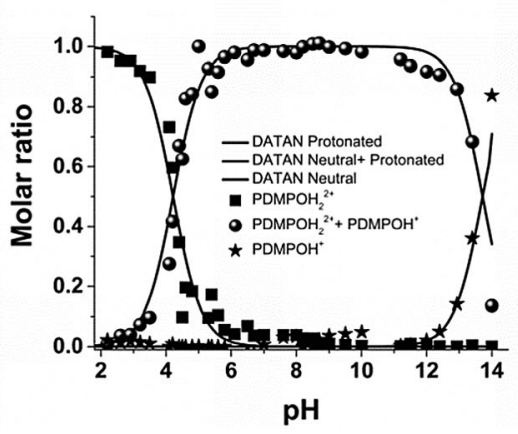

Fig. 3 (a) Fluorescence emission of PDMPO from $\mathrm{pH} 2.3$ to 14.0 using $360 \mathrm{~nm}$ excitation. (b) Spectra recovered from DATAN analysis suggested two forms, $\mathrm{PDMPOH}_{2}{ }^{2+*}$ and $\mathrm{PDMPOH}^{+*}$ with the behaviour between $\mathrm{pH}$ 4.2 and 13.7 due to excited state dynamics in the presence of water (see text for details). (c) Calculated molar ratios returned by DATAN suggest two $\mathrm{p} K_{\mathrm{a}}{ }^{*} \mathrm{~s}$ (4.2 and 13.7). The first of these corresponds to the point where ground state $\mathrm{PDMPOH}{ }^{+}$first appears while the second, $\mathrm{p} K_{\mathrm{w}}$, masks $\mathrm{p} K_{\mathrm{a} 1}$ * which is at least $9.5 \mathrm{p} K_{\mathrm{a}}$ units greater than $\mathrm{p} K_{\mathrm{a} 1}$ see text for details).

slight blue shift from the singly protonated species which was otherwise unobservable.

\subsection{PDMPO fluorescence in the presence of silica}

In contrast to aqueous phase fluorescence, DATAN analysis of PDMPO (Fig. 4a-c) in the presence of $50 \mathrm{~nm}$ silica particle retrieved similar spectroscopic species (Fig. 4a) but with a single $\mathrm{p} K_{\mathrm{a}}$ (10.4) (Fig. $4 \mathrm{~b}$ and $\mathrm{c}$ ) which we ascribe to $\mathrm{p} K_{\mathrm{a} 1}{ }^{*}$. The photoinduced proton transfer observed without silica particles is absent indicating the excited states do not have access to water in this environment (Scheme 3). However, the 


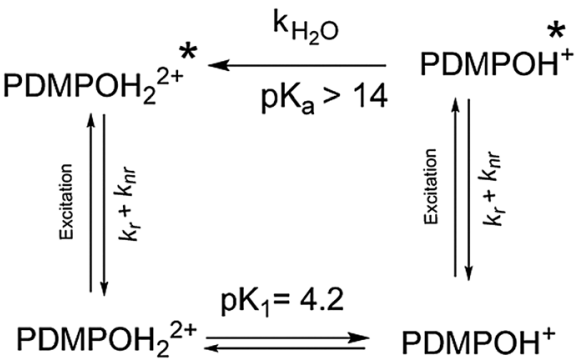

Scheme 2 Excited state behaviour of $\mathrm{PDMPOH}_{2}{ }^{2+*}$ and $\mathrm{PDMPOH}^{+*}$ in aqueous solution. Excitation of $\mathrm{PDMPOH}^{+}$leads to competition for $\mathrm{PDMPOH}^{+}$between extraction of a proton from water (represented by $k_{\mathrm{H}_{2} \mathrm{O}}$ ) and direct deactivation of the excited state (represented by the sum of the radiative $\left(k_{\mathrm{r}}\right)$ and non-radiative $\left(k_{\mathrm{n} r}\right)$ rate constants). The mixed form $(\mathrm{pH} 4.2$ to 13.7) results from competition kinetics between direct return to ground state $\mathrm{PDMPOH}^{+}$and excited state proton transfer from water to $\mathrm{PDMPOH}^{+*}$ forming $\mathrm{PDMPOH}_{2}{ }^{2+\star}$ followed by return to the ground state. A constant ratio is observed due to a constant value for $k_{\mathrm{H}_{2}} \mathrm{O}\left[\mathrm{H}_{2} \mathrm{O}\right]$.

Table 1 Solvent accessible surface area (SASA) for the three PDMPO species calculated from DFT and MD simulations

$\begin{array}{lllll}\frac{\text { PDMPO }}{\text { DFT MD }} & \frac{\text { PDMPOH }^{+}}{\text {DFT MD }} & \frac{\text { PDMPOH }_{2}^{2+}}{\text { DFT MD }}\end{array}$

\begin{tabular}{lllllll}
\hline SASA $\left(\AA^{2}\right)$ & 656.5 & $665.9 \pm 8.6$ & 651.8 & $659.9 \pm 6.9$ & 649.2 & $655.7 \pm 10.5$
\end{tabular}

spectra of both $\mathrm{PDMPOH}_{2}{ }^{2+}$ and $\mathrm{PDMPOH}^{+*}$ forms exhibit a marked $\mathrm{pH}$ dependent silica mediated chromaticity (Fig. 4a).
The protonated pyridinium form $\left(\mathrm{PDMPOH}_{2}{ }^{2+} ; ; \lambda_{\text {max,em }}>\right.$ $500 \mathrm{~nm}$ ) undergoes a blue shift as $\mathrm{pH}$ is increased up to $\mathrm{pH}$ ca. 9.3. The neutral pyridinium form $\left(\mathrm{PDMPOH}^{+} ; \lambda_{\mathrm{max}, \mathrm{em}}<\right.$ $460 \mathrm{~nm}$ ) undergoes a red shift as $\mathrm{pH}$ is increased from $c a .9 .7$ to 14. Further insight into the PDMPO-silica interaction was obtained by measuring the fluorescence anisotropy of PDMPO on silica particles from pH 2-14 (Fig. 4d). From pH 2.6-5.2, fluorescence anisotropy increases consistent with the ground state $\mathrm{p} K_{\mathrm{a} 1}$ (4.2). It reaches a plateau between $\mathrm{pH} 5.2$ and 8.2 indicating rotational diffusion more consistent with a larger particle. Above 8.2, the anisotropy decreases but remains slightly above that of the free dye up to $\mathrm{pH} 14$. This high $\mathrm{pH}$ transition on silica is consistent with the work of Ong et al. ${ }^{35}$ who observed a high $\mathrm{pH}$ species using second harmonic generation methods.

These data imply that at the high and low $\mathrm{pH}$ regimes there is single point binding of PDMPO via the side chain amine group giving the fluorogenic portion of the molecule the ability to undergo rotational diffusion. At intermediate $\mathrm{pH}$ the pyridine group is protonated and a transition to two point binding on the silica surface occurs resulting in hindered rotation. Under the experimental conditions used there is no evidence (Fig. 4c) that PDMPO is released from the surface, as there is no sign of the kinetically defined intermediate forms observed in aqueous solution (Fig. 4c).

To further understand PDMPO interactions with $50 \mathrm{~nm}$ diameter silica nanoparticles, the surface charge ( $)$ and hydrodynamic diameter $\left(D_{\mathrm{h}}\right)$ of the particles were measured before a

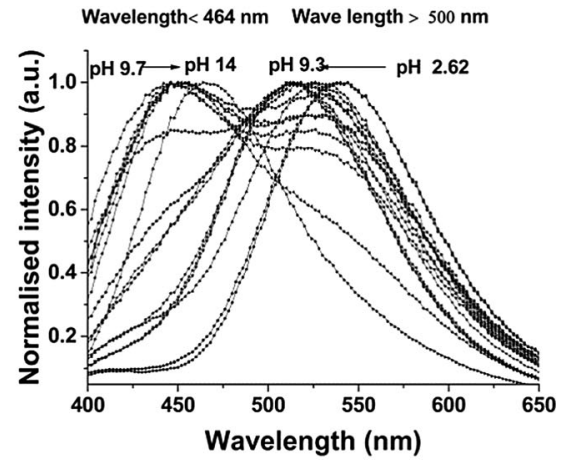

c

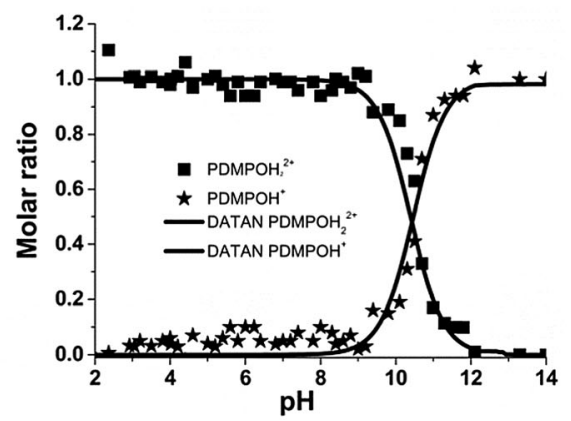

b

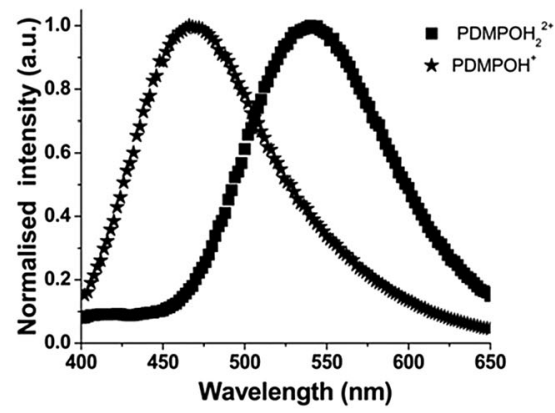

d

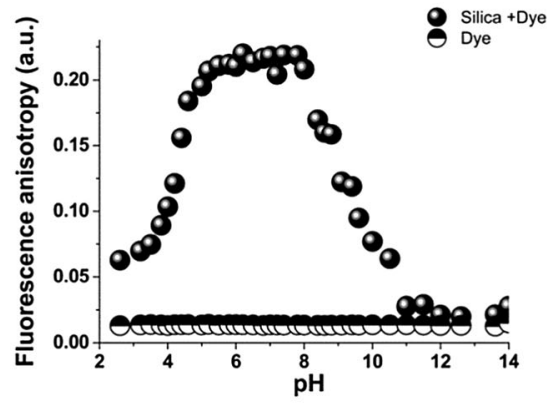

Fig. 4 PDMPO fluorescence over the range $\mathrm{pH} 2.6$ to 14.0 in the presence of $50 \mathrm{~nm}$ silica particles with excitation at $360 \mathrm{~nm}$ : (a) fluorescence emission spectra of PDMPO in the presence of silica; (b) calculated pure fluorescence spectra of $\mathrm{PDMPOH}_{2}{ }^{2+\star}$ and $\mathrm{PDMPOH}^{+\star}$ in the presence of silica from DATAN; (c) calculated molar ratio (recovered $\mathrm{p}_{\mathrm{a} 1}{ }^{*}=10.4$ ); (d) $\mathrm{pH}$ dependent fluorescence anisotropy of PDMPO in the presence and absence of $50 \mathrm{~nm}$ silica particles. 


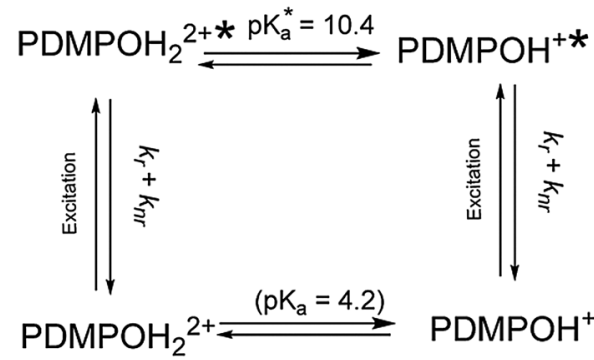

Scheme 3 Excited state behaviour of PDMPO in the presence of silica. In the presence of silica, the excited state $p K_{a}{ }^{*}$ is clearly visible and there is no evidence for excited state proton transfer from water. The ground state $\mathrm{p} K_{\mathrm{a}}(4.2)$ is assumed from the behaviour in the absence of SNPs.

and after interaction with the $0.1 \mu \mathrm{M}$ PDMPO (over a wide range of $\mathrm{pH}$ (1.0-12.0)) (Fig. 5a and b). The hydrodynamic diameter of the silica particles did not change significantly following interaction/adsorption of PDMPO over the $\mathrm{pH}$ range studied (Fig. 5a) implying that the dye does not extend beyond the Stern layer of the silica nanoparticles.

Addition of PDMPO did result in partial neutralization of the particles between $\mathrm{pH} 4.0$ and 9.5. This region coincides with the high anisotropy region suggesting that the first point of attachment via the amine residue is to both neutral silanol and siloxide groups while the second point of attachment via protonated pyridine brings a charge into the Stern-layer.

Further, PDMPO was added to $50 \mathrm{~nm}$ silica particles at neutral $\mathrm{pH}$ at a concentration of $1 \mathrm{mg} \mathrm{m}^{-1}$ and the concentration of PDMPO adjusted over the range 0.1 to $800 \mu \mathrm{M}$ (Fig. 5c). Increasing the PDMPO concentration resulted in the appearance of fluorescence consistent with aqueous PDMPO (unbound) and saturation of $\zeta$. This indicates that when PDMPO fluorescence is used with silica the concentration should be as low as possible $(0.1-5 \mu \mathrm{M})$ to maximise silicaphilic emission and minimize neutralization of the charge on the silica particles. Under ideal conditions, the amount of PDMPO should be $\leq 10 \%$ of the quantity required for full monolayer formation.

\subsection{Solvent effects on PDMPO fluorescence}

Although the spectra recovered by DATAN on silica and in the aqueous phase were nearly identical, there are $\mathrm{pH}$ dependent shifts in $\lambda_{\text {max,em }}$ of the two key forms of PDMPO on silica. These were most noticeable at low and intermediate $\mathrm{pH}$. To understand this behaviour, PDMPO was studied in a range of solvents and solvent mixtures (Fig. 6). PDMPO exhibited positive solvatochromism with the position of the neutral peak shifting over the range from $418 \mathrm{~nm}$ (chloroform, $\varepsilon_{\mathrm{r}}=4.8$ ) to $454 \mathrm{~nm}$ (water, $\varepsilon_{\mathrm{r}}=80.7$ ) (Fig. 6a). The position of the peak gives an estimate of the local dielectric constant (Fig. 6a and d) which when applied to $50 \mathrm{~nm}$ silica particles suggests the dielectric constant within the Stern layer surrounding the particles is close to that of water. Measurement of the spectroscopic response in water-acetonitrile and water-ethanol mixtures allowed us to assess the effects of dielectric constant on the form having a positively charged pyridine group and the distribution of neutral and positively a

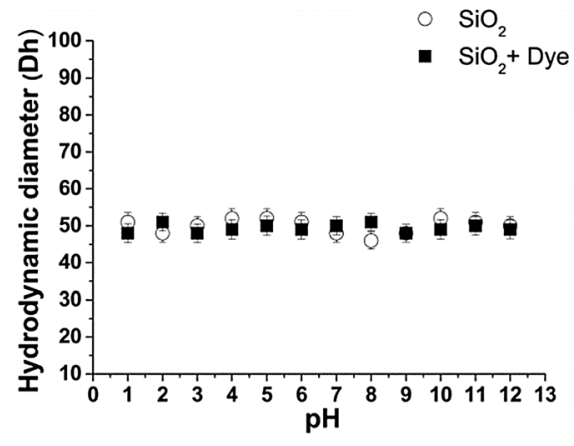

b

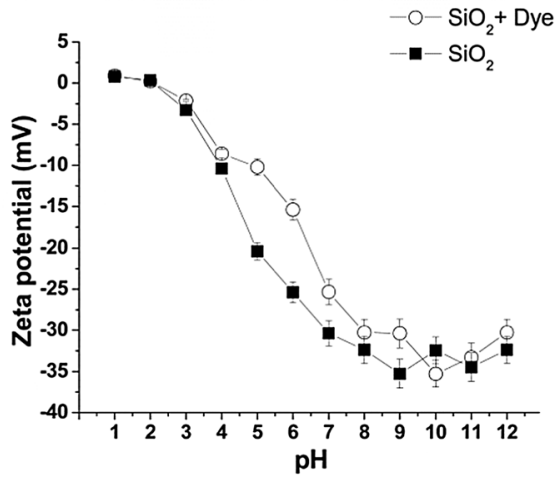

C

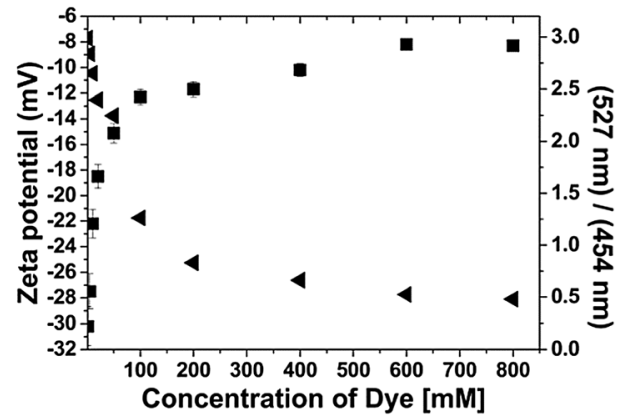

Fig. 5 (a) Effect of $\mathrm{pH}$ on the hydrodynamic diameter $\left(D_{h}\right)$ of Stöber particles with and without addition of PDMPO. (b) Effect of silica PDMPO interactions on zeta potential in the $\mathrm{pH}$ range 1.0 to 12.0 . (c) Dose dependent decrease in emission ratio $527 \mathrm{~nm} / 454 \mathrm{~nm}$ upon dye saturation on SNP surface $(50 \mathrm{~nm})$ ( $)$ and dose dependent decrease in zeta potential with increase in PDMPO concentration at $\mathrm{pH} 7.02$ for SNP of diameter $50 \mathrm{~nm}$ and concentration $1 \mathrm{mg} \mathrm{m}^{-1}(\varangle)$. The zeta potential of the silica $(50 \mathrm{~nm})$ alone was $-30 \mathrm{mV}$.

charged pyridine forms as the availability of protons from water decreases.

Although solvatochromism is a complex phenomen involving many interactions, ${ }^{36}$ these experiments yielded two conclusions. (i) PDMPO gave a clear solvatochromic shift in the neutral pyridine forms as the dielectric constant varied (Fig. 6d). Similar solvatochromism has been seen in other molecules and DFT studies suggest excited state dipole moments contribute to this. ${ }^{37}$ (ii) The yield of the neutral and $\mathrm{PDMPOH}^{+}$forms increased as the percentage of water decreased (Fig. $6 \mathrm{~b}$ and c), 


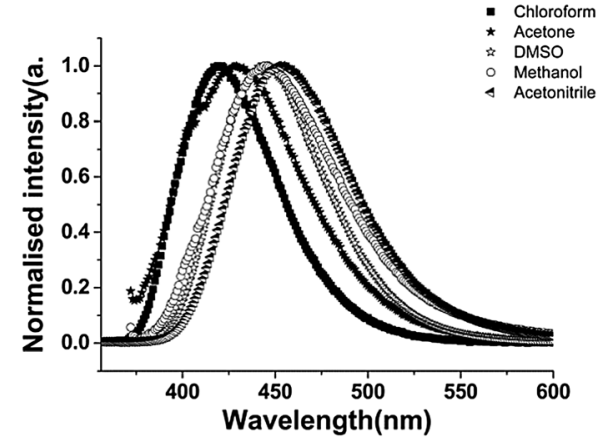

b

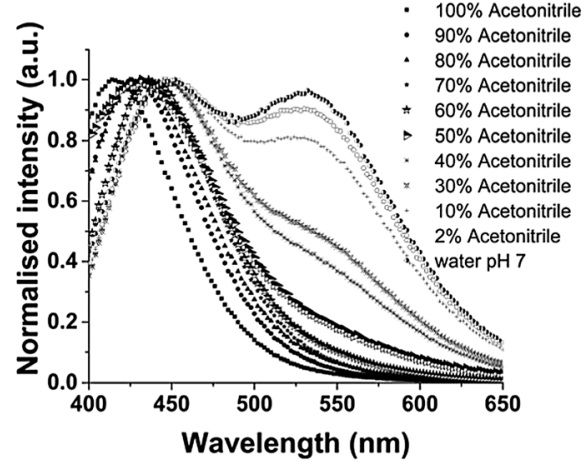

c

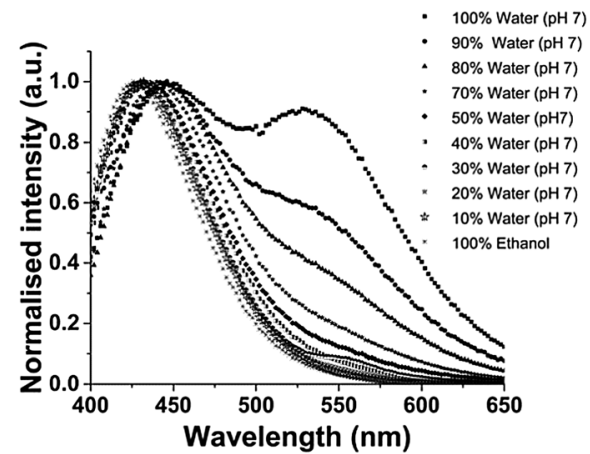

d

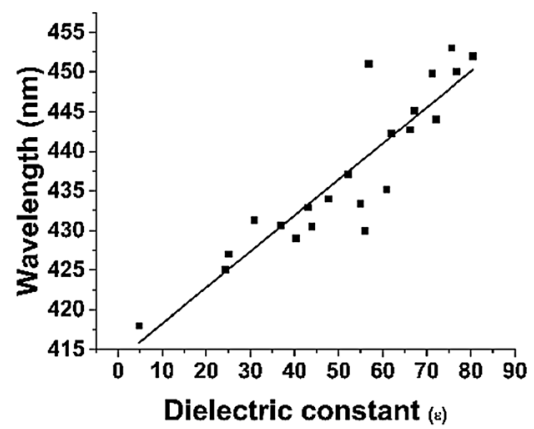

Fig. 6 PDMPO solvatochromism: (a) corrected emission spectra of neutral PDMPO in chloroform, acetone, DMSO, methanol and acetonitrile. Solvatochromism of PDMPO as the composition of solvent varied from 0 to $100 \%$ (v/v) for acetonitrile (b) and ethanol (c) and peak position with solution dielectric constant for these solutions (d).

even at $\mathrm{pH}$ 3.0, data not shown. These data are consistent with the interpretation presented in Scheme 2 and indicate that PDMPO is more sensitive to solvent than many other fluorescent probes. ${ }^{28}$ These effects indicate that, in addition to $\mathrm{pH}$, PDMPO reports on local dielectric constant and accessibility of water. a

b
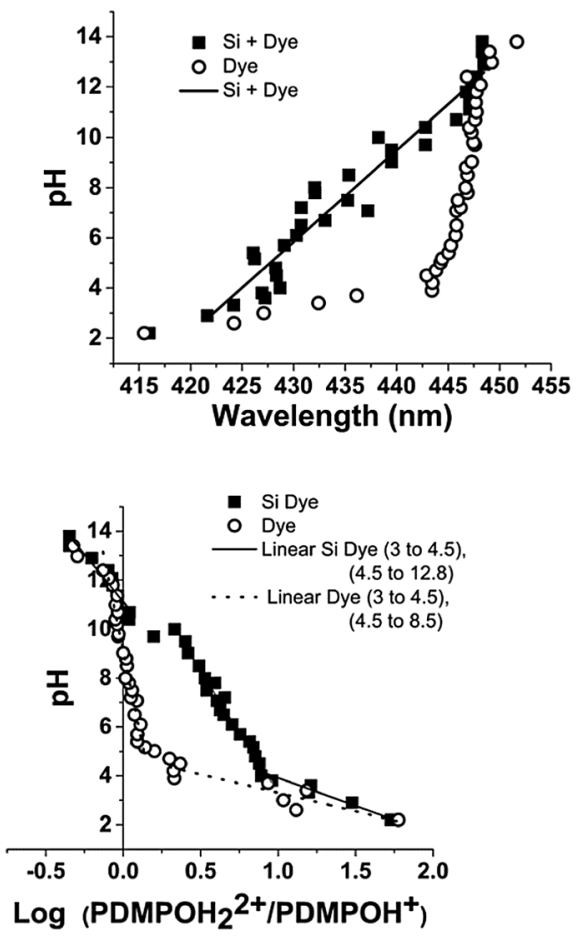

Fig. 7 Chromatic and ratiometric effects of $\mathrm{pH}$ on PDMPO in the presence ( $\mathbf{\square})$ and absence $(O)$ of silica nanoparticles: (a) chromatic shift in $\mathrm{PDMPOH}^{+\star}$ in the presence of silica allows estimation of $\mathrm{pH}$ and (b) ratiometric $\left(\log \left(\mathrm{PDMPOH}_{2}{ }^{+2 *} / \mathrm{PDMPOH}^{+}\right)\right)$estimation of $\mathrm{pH}$ in the presence of silica. Solid lines indicate useful regions for estimation of $\mathrm{pH}$. Dotted lines are to guide the eye.

\subsection{Indirect determination of $\mathrm{pH}$ using PDMPO-silica interactions}

The data in Fig. 4a and c suggest that below pH 8 PDMPO fluorescence cannot be used to monitor $\mathrm{pH}$ in the presence of silica as there is no applicable $\mathrm{p} K_{\mathrm{a}}{ }^{*}$. However, chromatic and ratiometric indicators (Fig. 7) show strong correlation with bulk $\mathrm{pH}$ in the presence of silica particles suspended in solution over the $\mathrm{pH}$ range $3-13$. The silica mediated shifts in PDMPO fluorescence emission maxima (Fig. 7a) arising from $\mathrm{pH}$ we ascribe to changes in dielectric constant at the silica surface.

This makes PDMPO chromaticity a proxy for bulk pH which extends the range over which this dye can sensibly be used to monitor pH (Fig. 7). The chromatic effects mask changes in the emission ratio during DATAN analysis (Fig. 7b). There is a continuous change in the ratio of the two forms over the entire range studied. At the lowest $\mathrm{pH}$, the basic form is not present and the ratio is affected by the Raman band of water which appears near the most shifted wavelengths for the basic form of the dye. Above pH 3-4 (the most useful pH's for silica studies) the behaviour of the ratio of the two forms appears to be mediated by silica in a way that affects both the ground and excited state. The dye alone shows more typical $\mathrm{pH}$ dependent behaviour with rapid changes consistent with the ground state $\mathrm{p} K_{\mathrm{a}}$ (4.2). As the $\mathrm{pH}$ increases, the ratio for the free dye is nearly constant until $\mathrm{p} K_{\mathrm{w}}$ is approached. This region is not seen in the silica bound dye. The free and silica bound forms coincide 
above $\mathrm{pH}$ 11. There is short plateau near $\mathrm{pH} 10$ for the silica bound dye which is believed to be due to the excited state $\mathrm{p} K_{\mathrm{a}}$. Sigma values from $\mathrm{PDMPOH}^{+}$and wavelength shift for $\mathrm{PDMPOH}_{2}{ }^{2+}$ can also be used as rough $\mathrm{pH}$ indicators (ESI $\dagger$ ). PDMPO is thus a unique molecule for interrogating $\mathrm{pH}$ and surface behaviour in the presence of silica. This response extends well beyond the range expected based on $\mathrm{p} K_{\mathrm{a}} \mathrm{s}$ in the absence of chromaticity.

\section{Discussion}

\subsection{Nature of PDMPO absorbance and fluorescence}

PDMPO is a probably the most common fluorescent dye used today to monitor silica deposition during biomineralization $^{28,29}$ and track the movement of lysosomes ${ }^{13}$ inside living cells. Nonetheless, the spectroscopic properties of the dye have been poorly understood until now. This study represents a significant advance in our understanding of both the aqueous and the silicaphilic fluorescence of PDMPO. The behaviour of PDMPO is similar to that of the fluorescent dyes 4-PYPO, 4-PYMPO, 4-PYMPOM, 2-PYMPO, 2-QUIMPO, 2-PYMPO- $\mathrm{CO}_{2} \mathrm{Me}$, and 2-PYMPO- $\mathrm{CH}_{2} \mathrm{OH} .{ }^{30}$ Photophysical properties of PDMPO most closely resemble that of the 4-PYMPO series, with $\mathrm{p} K_{\mathrm{a} 1} 4.2$ and 4.3 for PDMPO (current study) and PYMPO respectively. ${ }^{38} \mathrm{We}$ have shown for the first time that the fluorescence behaviour of the dye in aqueous solution above $\mathrm{pH} 4.2$ involves photo-induced proton transfer from water and/or the amine group to PDMPO which is disrupted when PDMPO binds to silica. While photoinduced proton transfer has been reported in the 4-PYMPO series of dyes, the disruption of this proton transfer by silica particles in aqueous solution has not been observed. The extensive chromaticity of PDMPO in the presence and absence of silica has not been previously documented nor has this been used previously as a proxy for bulk solution $\mathrm{pH}$ over the $\mathrm{pH}$ range 3-13. Similar chromaticity and/or disruption of photo-induced proton transfer in the presence of silica has not been reported for 4-PYMPO and related dyes, however, many will exhibit similar behaviour and screening related compounds for desirable silicaphilic fluorescence is likely to be fruitful.

\subsection{What is the nature of the silicaphilic behaviour of PDMPO?}

In the presence of silica, $\mathrm{p} K_{\mathrm{a} 1}{ }^{*}$ shifts $6.2 \mathrm{p} K_{\mathrm{a}}$ units and the photoinduced proton transfer observed in aqueous solution is disrupted. This indicates stabilisation of $\mathrm{PDMPOH}^{+}$through interaction with the silica surface and the absence of reactive water within its 'local' environment suggesting PDMPO does not extend beyond the Stern layer. PDMPO does not change the hydrodynamic diameter of the particles at any $\mathrm{pH}$ giving further evidence PDMPO is located within the Stern layer. This provides a model (Fig. 8) similar to ones arising from experiments investigating the interaction of cationic particles with ${ }^{39}$ large unilamellar vesicles (LUV). Since the silicaphilic response of the dye reports on the surface of the silica particles themselves, the concentration of the dye is critical. We suggest that

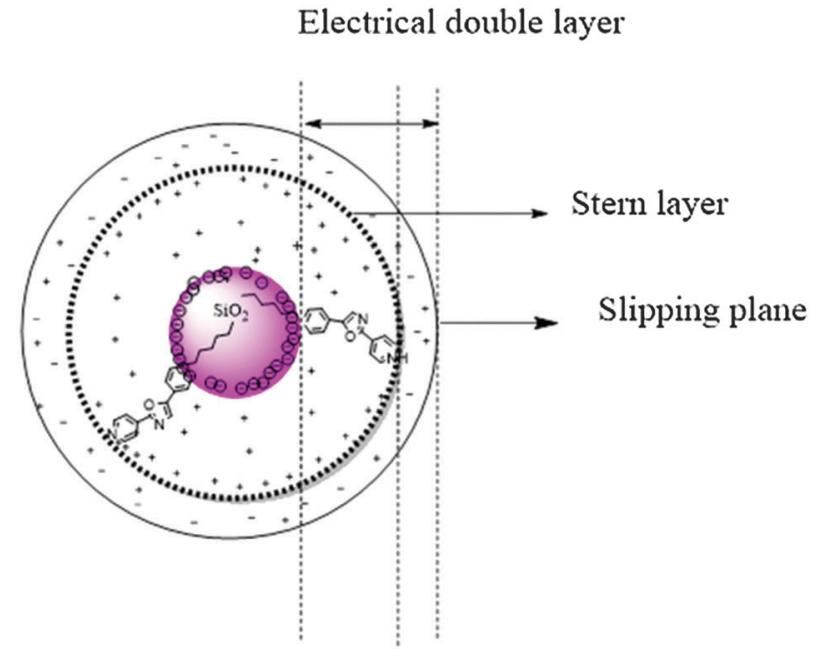

Fig. 8 Preferential location of PDMPO in the Stern layer.

dye concentrations be less than $10 \%$ of surface coverage to minimise fluorescence emission from the bulk solution.

\subsection{How does the dye bind to silica?}

The silicaphilic behaviour of PDMPO gives considerable insight into PDMPO-silica interactions. The fluorescence anisotropy data suggest that at all $\mathrm{pH}$ values below $c a .12$ the molecule is attached to the surface via either single point (low anisotropy) or multipoint modes (high anisotropy). Studies of electrostatically $v s$. tethered covalently bound dyes have shown the importance of electrostatic interactions in influencing the anisotropy of fluorophores on the silica surface. ${ }^{32}$ Our results confirm this while also making clear the role of $\mathrm{pH}$ on the charge states controlling the silica-dye interaction. We observed increases in the fluorescence anisotropy of PDMPO on silica above $\mathrm{p} K_{\mathrm{a} 1}$ which can only be explained by a change in the interaction resulting in more restrictive binding. We rationalise this with a two point binding model of PDMPO on silica (Fig. 9). We observed PDMPO partially neutralizing the surface charge of silica particles but only within a specific $\mathrm{pH}$ range (4.0-9.5) coinciding with the region of high fluorescence anisotropy. This indicates the pyridinium form is stabilised by interaction with negatively charged silica resulting in a proton being taken from the bulk solution. This proton is shared between a siloxide group and the pyridinium moiety on PDMPO leading to 2 point binding of PDMPO, loss of rotational diffusion (high anisotropy), and partial neutralisation of the silica surface. All the data reported here were taken at $\mathrm{pH}$ values above the point of zero charge for silica (ca. 2) and therefore all silica samples carry silanol and siloxide groups with their relative proportions changing with $\mathrm{pH}$. In all cases, adsorption of the dye can be attributed to electrostatic interaction mediated by protons between negatively charged silica surfaces and the dye. As the dye is held in close proximity to the silica surface, it is highly sensitive to $\mathrm{pH}$ dependent changes in local dielectric constant which gives rise to the observed shifts in emission maxima. 

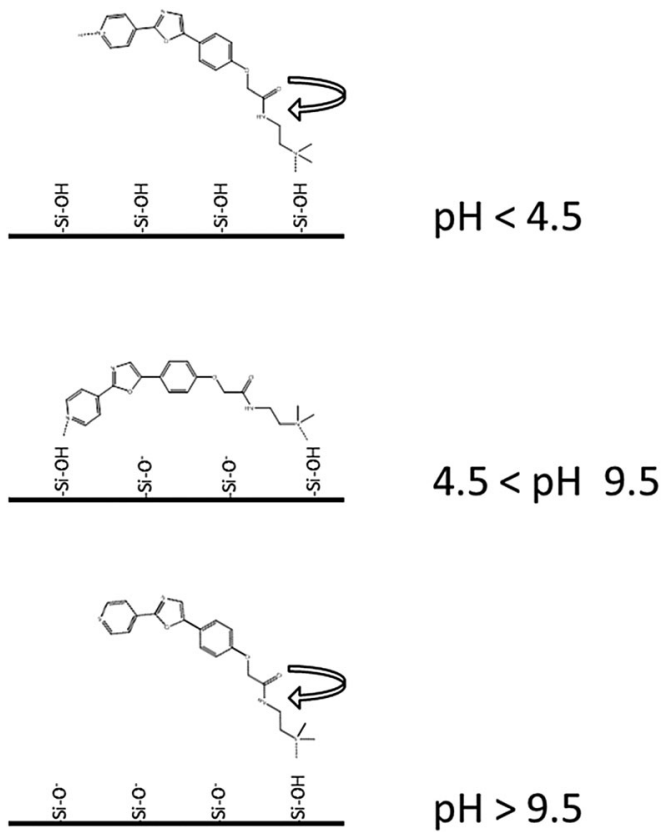

Fig. $9 \mathrm{pH}$ dependent binding of PDMPO on silica surface the arrow represents depolarising motion.

\subsection{PDMPO-silica interactions: an indirect measure of solution $\mathbf{p H}$}

The data presented in Fig. 7 show that PDMPO interacting with silica can be used as a probe of bulk $\mathrm{pH}$ over a wide $\mathrm{pH}$ range (ca. 3-13). However, the relevant ground and excited state $\mathrm{p} K_{\mathrm{a}} \mathrm{s}$ (4.2 and 10.4) are insufficient to cover such a wide range of $\mathrm{pH}$. Outside of these regions, the chromatic behaviour serves as a proxy for $\mathrm{pH}$. It is clear that the presence of silica is critical and the data show that there is a relationship between $\mathrm{pH}$ and local dielectric constant when silica is present. Unpicking the various contributions to the observed behaviour remains to be done.

\section{Conclusions and future work}

PDMPO has been widely used in vivo to study new silica deposition. Examples include: precipitation of silica in diatoms, ${ }^{2,3}$ silica deposition in Equisetum arvense, ${ }^{4}$ identifying silica transporters in silicifying organisms ${ }^{5}$ such as Bolidophyceae and Parmales. ${ }^{6}$ The more detailed understanding provided by the current study opens up many new applications. The ability to directly report on $\mathrm{pH}$ within biological organelles over an unprecedented range with a single dye will allow us to monitor the formation of silica more effectively, both in vitro and in vivo. This will extend our knowledge of the fundamentals of silica formation in the laboratory and in organisms, such as diatoms and may allow us to follow silica formation in the silica deposition vesicle which has not been possible. It will also allow us to probe individual silica structures within composites of mixed silica structures produced by silicifying organisms including single celled organisms such as diatoms and multicellular plants and animals such as sponges. PDMPO may also be used more widely to quantitatively investigate biophysical interactions of silica at solid liquid interfaces, colloid stabilization, and the assembly of silica thin films and to study electrostatic interactions between polyelectrolytes on thin films made of silica.

\section{Acknowledgements}

CCP and MP are grateful for the funding of a PhD studentship from NTU. AFOSR, through grants FA9550-10-1-0024 and FA9550-13-1-0040 are acknowledged for their funding of instrumentation. FMM, TG and MJB acknowledge funding from AFOSR (FA9550-11-1-0199) and ONR-PECASE (N00014-10-1-0562).

\section{References}

1 K. Shimizu, Y. Del Amo, M. A. Brzezinski, G. D. Stucky and D. E. Morse, Chem. Biol., 2001, 8, 1051.

2 C. A. Durkin, A. Marchetti, S. J. Bender, T. Truong, R. Morales, T. Mock and E. V. Armbrust, Limnol. Oceanogr., 2012, 57, 1619.

3 S. Hazelaar, H. J. van der Strate, W. W. C. Gieskes and E. G. Vrieling, J. Phycol., 2005, 41, 354.

4 C. Law and C. Exley, BMC Plant Biol., 2011, 11, 112.

5 K. Ogane, A. Tuji, N. Suzuki, A. Matsuoka, T. Kurihara and R. S. Hori, Mar. Micropaleontol., 2010, 77, 137.

6 M. Ichinomiya, S. Yoshikawa, M. Kamiya, K. Ohki, S. Takaichi and A. Kuwata, J. Phycol., 2011, 47, 144-151.

7 M. Kucki and T. Fuhrmann-Lieker, J. R. Soc., Interface, 2012, 9, 727.

8 C. W. Li, S. Chu and M. Lee, Protoplasma, 1989, 151, 158.

9 C. Perry, E. Moss and R. Williams, Proc. R. Soc. B, 1990, 241, 47.

10 M. J. Hodson, R. J. Smith, A. Vanblaaderen, T. Crafton and C. H. Oneill, Ann. Occup. Hyg., 1994, 38, 149.

11 Z. J. Diwu, C. S. Chen, C. L. Zhang, D. H. Klaubert and R. P. Haugland, Chem. Biol., 1999, 6, 411.

12 Z. Diwu, Y. X. Lu, C. L. Zhang, D. H. Klaubert and R. P. Haugland, Photochem. Photobiol., 1997, 66, 424.

13 S. Hurwitz, M. Terashima, N. Mizunuma and C. Slapak, Blood, 1997, 89, 3745.

14 H. M. DePedro and P. Urayama, Anal. Biochem., 2009, 384, 359.

15 C. A. Durkin, A. Marchetti, S. J. Bender, T. Truong, R. Morales, T. Mock and E. V. Armbrust, Limnol. Oceanogr., 2012, 57, 1619.

16 M. Ichinomiya, Y. Gomi, M. Nakamachi, T. Ota and T. Kobari, Deep Sea Res., Part II, 2010, 57, 1665.

17 M. A. Saxton, N. A. D'souza, R. A. Bourbonniere, R. M. L. McKay and S. W. Wilhelm, J. Great Lakes Res., 2012, 38, 206.

18 S. H. Hilal, S. W. Karickhoff and L. A. Carreira, Quant. Struct.-Act. Relat., 1995, 14, 348.

19 J. E. Leffler and E. Grunwald, Rates and equilibria of organic reactions as treated by statistical, thermodynamic and extrathermodynamic methods, Wiley, New York, 1963 (Dover reprint, 2013). 
20 L. P. Hammett, Physical Organic Chemistry, McGraw Hill, New York, 1970.

21 M. J. S. Dewar, The Molecular Orbital Theory of Organic Chemistry, McGraw Hill, New York, 1969.

22 F. Neese, Wiley Interdiscip. Rev.: Comput. Mol. Sci., 2012, 2, 73.

23 A. D. Becke, J. Chem. Phys., 1993, 98, 5648.

24 C. T. Lee, W. T. Yang and R. G. Parr, Phys. Rev. B: Condens. Matter Mater. Phys., 1988, 37, 785.

25 P. Geerlings, F. De Proft and W. Langenaeker, Chem. Rev., 2003, 103, 1793.

26 S. Plimpton, J. Comput. Phys., 1995, 117, 1.

27 W. Humphrey, A. Dalke and K. Schulten, J. Mol. Graphics, 1996, 14, 33.

28 I. Scarminio and M. Kubista, Anal. Chem., 1993, 65, 409-416.

29 A. Elbergali, J. Nygren and M. Kubista, Anal. Chim. Acta, 1999, 379, 143.

30 J. Lakowicz, Principles of Fluorescence Spectroscopy, Springer, USA, 2006.
31 P. Roach, D. Farrar and C. Perry, J. Am. Chem. Soc., 2006, 128, 3939.

32 J. Faver and K. M. Merz Jr., J. Chem. Theory Comput., 2010, 6, 548.

33 Z. J. Diwu, C. S. Chen, C. L. Zhang, D. H. Klaubert and R. P. Haugland, Chem. Biol., 1999, 6, 411.

34 N. Mataga, Y. Kaifu and M. Koizumi, Bull. Chem. Soc. Jpn., 1956, 29, 465.

35 S. W. Ong, X. L. Zhao and K. B. Eisenthal, Chem. Phys. Lett., 1992, 191, 327.

36 A. Marini, A. Munoz-Losa, A. Biancardi and B. Mennucci, J. Phys. Chem. B, 2010, 114, 17128.

37 A. Amat, C. Miliani, A. Romani and S. Fantacci, Phys. Chem. Chem. Phys., 2015, 17, 6374.

38 S. Charier, O. Ruel, J. B. Baudin, D. Alcor, J. F. Allemand, A. Meglio, L. Jullien and B. Valeur, Chem. - Eur. J., 2006, 12, 1097.

39 D. de Sousa Neto, A. Hawe and M. Tabak, Eur. Biophys. J. Biophys. Lett., 2013, 42, 267. 